\title{
GEOMETRIC OBJECTS DEFINED BY ALMOST LIE STRUCTURES
}

\author{
MARCELA POPESCU and PAUL POPESCU \\ P.O. Box 4-66, Craiova 1100, Romania \\ E-mail: marcelapopescu@email.com, paulpopescu@email.com
}

\begin{abstract}
The aim of this paper is to extend from manifolds to vector bundles some classical geometric objects, associated with Lagrange and Hamilton metrics. Considering vector bundles endowed with almost Lie structures, defined in [24] by one of the authors, some geometric objects like R-(semi)sprays and R-connections of Cartan type are defined and studied. It is proved that the Lagrange equations deduced for Lie algebroids by A. Weinstein have a similar form for almost Lie structures.
\end{abstract}

1. Introduction. An anchored vector bundle (AVB) (or a relative tangent space in $[23,26])$ is a couple $(\theta, D)$, where $\theta=(R, q, M)$ is a vector bundle and $D: \theta \rightarrow \tau M$ is a vector bundle morphism called an anchor (an arrow, or a tangent map), where the vector bundle $\tau M=(T M, p, M)$ is the tangent bundle of $M$.

A Lie algebroid on a vector bundle $\theta$ with a skew symmetric bracket $[\cdot, \cdot]_{\theta}: \Gamma(\theta) \times$ $\Gamma(\theta) \rightarrow \Gamma(\theta)$ on the module of sections is the most known situation involving an AVB. The bracket of a Lie algebroid must verify some restrictive conditions, such as: the Leibniz identity, the compatibility with the Lie bracket and the Jacobi identity. If the last two conditions are not fulfilled by a bracket on an AVB, then, following [24], the triple $\left(\theta, D,[\cdot, \cdot]_{\theta}\right)$ is called an almost Lie structure $(A L S)$. An interpretation of an ALS is given in [24], where it is proved that it is a one to one correspondence between ALS's and 1-degree derivations on the exterior algebra of skew symmetric forms on $\theta$. In this order of ideas, a Lie algebroid corresponds to a 1-degree derivation with a null square (see also [34, Lemma 2.2]).

In the case when the bracket of an ALS verifies all the conditions of a Lie algebroid, except the Jacobi identity, the ALS is called an algebroid. Generalized algebroids with arbitrary brackets (when the Leibniz' and Jacobi's conditions are not fulfilled) are studied in [27]. The Courant algebroids studied in [11] are particular cases of generalized algebroids. The role played by the Jacobi identity in the case of Lie algebras and Lie algebroids is underlined in [3].

2000 Mathematics Subject Classification: 53B15, 55R10, 55R25, 53C07, 22A30.

The paper is in final form and no version of it will be published elsewhere. 
If $\theta$ is an arbitrary vector subbundle of $\tau M$ (i.e. a geometric regular distribution) and $i: \theta \rightarrow \tau M$ is the inclusion morphism, then the $\operatorname{AVB}(\theta, i)$ is a non-holonomic space of $\mathrm{G}$. Vrănceanu ([32]). This non-holonomic space was the background to consider geometric objects related with mechanics, differential equations etc. The modern sub-Riemannian geometry studies also the non-integrable regular distributions, but a metric tensor on the fibers of the distribution is also given (see, for example, [30, 19]). Many problems which arise in the sub-Riemannian geometry (for example in [30]) may be considered in a more general situation of an arbitrary AVB.

The AVB is the background of linear pseudoconnections of Wong [33], which generalize the usual linear connections. The non-linear R-connections introduced in [23] and called below R-connections can be interpreted as (non-)linear pseudoconnections of Wong. Moreover, a bracket on an AVB (i.e. an ALS) enables to consider also the curvature of an R-connection (see [24]). In the particular case of Lie algebroids, the linear R-connections (respectively linear pseudoconnections of Wong) are just the A-connections defined by Fernandez in [4].

An R-(semi)spray is defined and it is interpreted in this paper as a second order differential equation (SODE) on an ALS. The particular case of an R-spray (using another equivalent definition) and of an injective anchor (i.e. when $\theta$ is isomorphic with a vector subbundle of $\tau M$ ) is considered in [29], where the author generalizes a result of AmbroseSinger-Palais, proving that every R-spray can be associated with a linear connection on $\theta$. In the last section this result is extended for every R-spray which is not necessary differentiable on the image of the null section.

From the algebraic view point, AVB's correspond to finitely generated modules of vector fields (a submodule $\mathcal{M} \subset \mathcal{X}(M)$ is finitely generated iff there is an $\operatorname{AVB}(\theta, D)$ over the base $M$ such that $\mathcal{M}=D(\Gamma(\theta)))$. When $\mathcal{M}$ has a non-constant rank, then the $\operatorname{AVB}(\theta, D)$ can be considered as a desingularization of $\mathcal{M}$.

The case of an infinite dimensional AVB is studied in [20], where it is proved that every AVB defines a smooth distribution on the base manifold. As in the case of a finite dimensional manifold, this distribution is generally non-regular (i.e. it is not defined by a vector subbundle of the tangent bundle). In our paper we deal only with the finite dimensional manifolds and bundles, but most of our constructions work in the infinite dimensional case, too.

The aim of this paper is to show how the classical lagrangian and hamiltonian formalism can be extended and developed for ALS's, pointing out some aspects based on presymplectic forms, exterior calculus, second order differential equations and (non-linear) connections considered in this frame. Notice that the lagrangian formalism on Lie algebroids is described in [35] (related especially to Lie groupoids) and in [10].

In the second section we start from [23, 24], where it is shown that a given AVB or an ALS can be used as a substitute of the tangent bundle of the base. Some adapted ALS's are considered and some presymplectic forms and symplectic connections (used in the next sections) are studied.

In the third section we show that a Cartan-Kern R-connection can be associated with a lagrangian on an ALS. This connection is a symplectic one and it corresponds to itself when Proposition 2.4 is applied. We define also the Legendre transformation and we show 
that its R-differential is a symplectic morphism, which sends the Kern-Cartan connection to a symplectic R-connection on the dual.

In the fourth section, a physical interpretation concerning the constructions performed in the previous sections is given. Using the formalism presented in this paper, we prove that the Lagrange equations for a Lie algebroid, given in [35], keep the same form in the general case of an ALS. In order to emphasize the link between the two approaches, two different proofs of the Lagrange equations are given: the first is a direct one, using the previous constructions, and the second one follows the Weinstein's ideas, using an almost Lagrange Poisson bracket (see [35]). Thus, our construction is a natural extension of the classical case and of the Lie algebroids case, too.

A result from [29], which, in turn, generalizes a theorem of Ambrose-Palais-Singer, is extended in the last section. It refers to the possibility to define an R-spray using a suitable homogeneous non-linear connection, in the case when the anchor is injective on fibres. In [29] the R-spray $S$ (called a spray-like) is differentiable on the entire $R$, while in our paper $S$ is differentiable on $\tilde{R}=R \backslash\{0\}$.

Notice that, in order to simplify the exposition, we use often local coordinates. In spite of this fact, all the objects have global definitions. All the differentiable structures considered below are of class $C^{\infty}$.

2. Lagrangian and hamiltonian geometry on anchored vector bundles. In this section we start from [23, 24], where it is shown that a given AVB or an ALS can be used as a substitute of the tangent bundle of the base. Some adapted ALS's are considered and some presymplectic forms and symplectic connections (used in the next sections) are studied.

Let $(\theta, D)$ be an arbitrary AVB and $\xi=(E, \pi, M)$ be another vector bundle on the same base $M$. Let $R E=T E \times_{T M} R=\left\{(x, y) \in T E \times R: \pi_{*}(x)=D(y)\right\}$ be the fibered product of the differential tangent map $\pi_{*}: T E \rightarrow T M$ and the given anchor $D: R \rightarrow \tau M$. Let $\Delta: R E \rightarrow T E$ be the canonical projection, $\bar{\pi}$ the canonical projection of the tangent bundle $\tau E$ and $s=\bar{\pi} \circ \Delta$. Then the fibered manifold $R \xi=(R E, s, E)$ is a vector bundle, $\Delta$ is an anchor on $R \xi$ (called the canonical anchor) and $(R \xi, \Delta)$ is an AVB, called the $R-A V B$ of $\xi$. The diagram:

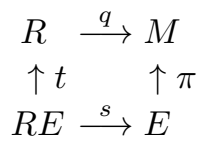

commutes; it has all the arrows as projections of vector bundles, the arrow $t$ is a $\pi$ morphism and the arrow $s$ is a $q$-morphism of vector bundles. Notice that the diagram (1) implies that $(R E, R, E)$ is a double vector bundle (see $[9,5])$. If $\xi=\theta$, then $(R R, R, R)$ becomes also a double vector bundle.

Notice that the couple $(R \xi, \Delta)$ is an AVB, too.

The $R$-vertical bundle of $\xi$ related to the $\operatorname{AVB}(\theta, D)$ is the vector subbundle ker $t \stackrel{\text { not. }}{=}$ $V R \xi \subset R \xi$. Since $V R \xi$ is canonically isomorphic with the vertical bundle $V \xi$, we identify $V R \xi$ with $V \xi$. A non-linear $R$-connection (or simply an $R$-connection) on $\xi$ related to the $\operatorname{AVB}(\theta, D)$ is a left splitting of the inclusion morphism $i: V \xi \rightarrow R \xi$, i.e. a vector bundle 
morphism $C: R \xi \rightarrow V \xi$ such that $C \circ i=i d_{V \xi}$. Since $C$ is a surjection, there is a vector subbundle $\operatorname{ker} C \stackrel{\text { not. }}{=} H \xi$, called the $R$-horizontal bundle of $\xi$, which is supplementary to $V \xi$ in $R \xi$. Thus, to give an R-connection $C$ on $\xi$ is equivalent to give a vector subbundle $H \xi \subset R \xi$ which is supplementary to $V \xi$.

A linear R-connection (or a linear pseudoconnection of Wong [33]) on the vector bundle $\xi$ related to the AVB $(\theta, D)$ is a map $\nabla: \Gamma(\theta) \times \Gamma(\xi) \rightarrow \Gamma(\xi)$ such that the Koszul conditions hold. As it is shown in $[23,25]$, a linear R-connection defines a homogeneous R-connection.

Notice that (1) is a natural generalization of the diagram:

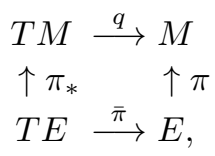

where the bottom and the top arrows were replaced by the canonical projections of certain anchored vector bundles. This allows us to reconsider most of the basic constructions related to tangent bundles.

A bracket (or a Lie map) on an $\operatorname{AVB}(\theta, D)$ is a map $[\cdot, \cdot]_{\theta}: \Gamma(\theta) \times \Gamma(\theta) \rightarrow \Gamma(\theta)$ which is bilinear over $\mathbb{R}$, skew symmetric and $[X, f Y]_{\theta}=(D X)(f) Y+f[X, Y]_{\theta},(\forall) X, Y \in \Gamma(\theta)$, $f \in \mathcal{F}(M)$. The triple $\left(\theta, D,[\cdot, \cdot]_{\theta}\right)$ is an almost Lie structure (ALS). The construction of the exterior differential calculus on an ALS is performed in [24], where it is also proved that there is a one to one correspondence between the ALS's on a vector bundle $\theta$ and the 1-degree derivations of the exterior algebra of the dual bundle $\theta^{*}$. The 1 -differential $d_{\theta}$ which corresponds to the $\operatorname{ALS}\left(\theta, D,[\cdot, \cdot]_{\theta}\right)$ is given by the following formula:

$$
\begin{gathered}
d_{\theta} \omega\left(X_{0}, \ldots, X_{p}\right)=\sum_{i=0}^{p}(-1)^{i}\left(D X_{i}\right)\left(\omega\left(X_{0}, \ldots, \widehat{X}_{i}, \ldots, X_{p}\right)\right)+ \\
\sum_{0 \leq i<j \leq p}(-1)^{i+j} \omega\left(\left[X_{i}, X_{j}\right]_{\theta}, X_{0}, \ldots, \widehat{X}_{i}, \ldots, \widehat{X}_{j}, \ldots, X_{p}\right), \\
(\forall) X_{0}, \ldots, X_{p} \in \Gamma(\theta), \omega \in \mathcal{A}^{p}(\theta), \text { for } p \geq 0,
\end{gathered}
$$

where $\mathcal{A}^{p}(\theta)$ is the exterior algebra of skew symmetric forms $\omega: \underbrace{\Gamma(\theta) \times \cdots \times \Gamma(\theta)}_{p \text { times }} \rightarrow$ $\mathcal{F}(M)$. In the particular case $p=0$, we have $d_{\theta} f(X)=(D X)(f),(\forall) X \in \Gamma(\theta), f \in$ $\mathcal{F}(M)=\mathcal{A}^{0}(\theta)$.

An algebroid is an ALS $\left(\theta, D,[\cdot, \cdot]_{\theta}\right)$ provided that $[D X, D Y]=D\left([X, Y]_{\theta}\right),(\forall) X, Y \in$ $\Gamma(\theta)$, where the first bracket is the Lie bracket on $\mathcal{X}(M)$. A Lie algebroid is an algebroid $\left(\theta, D,[\cdot, \cdot]_{\theta}\right)$ such that the Jacobiator is null:

$$
\mathcal{J}(X, Y, Z) \equiv \sum_{\text {cycl. }}\left[\left[X, Y_{\theta}\right], Z\right]_{\theta}=0,(\forall) X, Y, Z \in \Gamma(\theta) .
$$

Notice that an $\operatorname{ALS}\left(\theta, D,[\cdot, \cdot]_{\theta}\right)$, which defines the 1 -derivation $d_{\theta}$, is:

- an algebroid iff $d_{\theta}^{2} f=0,(\forall) f \in \mathcal{F}(M)$;

- a Lie algebroid iff $d_{\theta}^{2} f=0$ and $d_{\theta}^{2} \omega=0,(\forall) f \in \mathcal{F}(M), \omega \in \mathcal{A}^{1}(\theta)$, i.e. $d_{\theta}^{2}=0$.

But, for an arbitrary ALS, we have to remark that $d_{\theta}^{2} \neq 0$. 
Let $\left(\theta, D,[\cdot, \cdot]_{\theta}\right)$ be a given ALS and consider the AVB $(\theta \wedge \theta, \mathcal{D})$, where $\mathcal{D}(X \wedge Y)=$ $[D(X), D(Y)]-D\left([X, Y]_{\theta}\right),(\forall) X, Y \in \Gamma(\theta)$. Let $\xi=(E, \pi, M)$ be a vector bundle (or, generally, a fibered manifold) over the same base. If $\left\{L_{\beta \gamma}^{\alpha}\right\}$ are the components of the bracket $[\cdot, \cdot]_{\theta}$ in a local base of the sections of $\theta$ and $\left\{\bar{L}_{\beta \gamma}^{a}\right\}$ are the components of an R-connection on $\xi$, related to the AVB $(\theta \wedge \theta, \mathcal{D})$, then $\left\{\bar{L}_{\beta \gamma}^{\alpha}=L_{\beta \gamma}^{\alpha}, \bar{L}_{\beta \gamma}^{a}, \bar{L}_{\beta c}^{a}=\bar{L}_{b c}^{a}=\right.$ $\left.\bar{L}_{\beta c}^{\alpha}=\bar{L}_{b c}^{\alpha}=0\right\}$ are the components of an adapted bracket $[\cdot, \cdot]_{R \xi}$ on $R \xi$. The ALS $\left(R \xi, \Delta,[\cdot, \cdot]_{R \xi}\right)$ on $R \xi$ is called adapted. (See $[24,25]$ for more details.) In the particular case of an algebroid (or a Lie algebroid), $\mathcal{D}=0$, thus $\left\{\bar{L}_{\beta \gamma}^{a}\right\}$ are the components of a mixed tensor and can be taken (canonically) zero.

In the sequel, using ALS's, we extend the canonical symplectic form on the cotangent space of a manifold to a presymplectic form on $R \theta^{*}$.

In this order of ideas, we recall some definitions. Given a vector bundle, one says that a non-degenerate bilinear 2-form on the fibers is a Leibniz form and a skew symmetric Leibniz form on the fibers is a presymplectic form; the vector bundle is called a symplectic vector bundle (a Leibniz vector bundle) whenever it has a presymplectic form (a Leibniz form) on fibers.

We start from a given AVB $(\theta, D)$, where $\theta=(R, q, M)$, and we consider the Ranchored vector bundle $R \theta^{*}=\left(R R^{*}, s^{*}, R^{*}\right)$ of the dual bundle $\theta^{*}$. In this case the diagram (1) becomes:

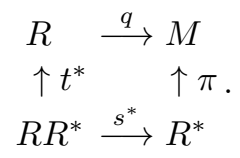

A canonical 1-form $\omega \in \Gamma\left(R^{*} \theta^{*}\right)=\mathcal{A}^{1}\left(R \theta^{*}\right)$, where $R^{*} \theta^{*}=\left(R \theta^{*}\right)^{*}$, can be defined using the formula $\omega_{u}\left(X_{u}\right)=u\left(t_{u}^{*}\left(X_{u}\right)\right),(\forall) u \in R^{*}, X_{u} \in R R_{u}^{*}$.

Using this canonical 1-form and an adapted ALS on $R \theta^{*}$ we prove the following result.

Proposition 2.1. If $\left(\theta, D,[\cdot, \cdot]_{\theta}\right)$ is an $A L S$, then the 2 -form $\Omega=d_{R \theta^{*}} \omega \in \mathcal{A}^{2}\left(R \theta^{*}\right)$ does not depend on the adapted $A L S$ on $R \theta^{*}$ and it is non-degenerate, thus $\left(R \theta^{*}, \Omega\right)$ is a symplectic vector bundle.

Proof. In an analogous way as in [24], we use the adapted local coordinates: $\left(x^{i}\right)$ on $M,\left(x^{i}, X^{\alpha}\right)$ on $R,\left(x^{i}, p_{\alpha}\right)$ on $R^{*},\left(x^{i}, p_{\alpha}, X^{\beta}, P_{\gamma}\right)$ on $R R^{*}$ and $\left(x^{i}, p_{\alpha}, U_{\beta}, Q^{\gamma}\right)$ on $R^{*} R^{*}$. We denote by $\left\{\omega^{\beta}, S_{\gamma}\right\}$ and $\left\{s_{\alpha}\right\}$ the local bases of $\Gamma\left(R^{*} \theta^{*}\right)$ and $\Gamma(\theta)$ respectively, which correspond to the above coordinates and by $\left\{L_{\alpha \beta}^{\gamma}\right\}$ the local functions on $M$ defined by the bracket on $\theta:\left[s_{\alpha}, s_{\beta}\right]_{\theta}=L_{\alpha \beta}^{\gamma} s_{\gamma}$. Then, using the local base $\left\{d_{R \theta^{*}} p_{\alpha}, \omega^{\beta}\right\}$ of $\mathcal{A}^{1}\left(R \theta^{*}\right)$, $\omega$ has the local form $\omega_{\left(x^{i}, p_{\alpha}\right)}=p_{\alpha} \omega^{\alpha}$ and formula (3) gives $d_{R \theta^{*}} \omega^{\alpha}=-\frac{1}{2} L_{\beta \gamma}^{\alpha} \omega^{\beta} \wedge \omega^{\gamma}$. It follows that

$$
\Omega=d_{R \theta^{*}} \omega=d_{R \theta^{*}} p_{\alpha} \wedge \omega^{\alpha}+p_{\alpha} d_{R \theta^{*}} \omega^{\alpha}=d_{R \theta^{*}} p_{\alpha} \wedge \omega^{\alpha}-\frac{1}{2} p_{\alpha} L_{\beta \gamma}^{\alpha} \omega^{\beta} \wedge \omega^{\gamma},
$$

thus $\Omega$ is non-degenerate, since $\left\{d_{R \theta^{*}} p_{\alpha} \wedge d_{R \theta^{*}} p_{\beta}, d_{R \theta^{*}} p_{\alpha} \wedge \omega^{\beta}, \omega^{\alpha} \wedge \omega^{\beta}\right\}$ is a local base in $\mathcal{A}^{2}\left(R \theta^{*}\right)$.

In the particular case $\theta=\tau M$ we obtain that $\omega=p_{i} d x^{i} \in \Gamma\left(\tau^{*} T^{*} M\right)=\mathcal{X}^{*}\left(T^{*} M\right)$ is the canonical 1-form on the vector bundle $\tau T^{*} M$. Then $\Omega=d \omega$ is the canonical symplectic structure on $T^{*} M$, which defines the well-known Poisson structure on $T^{*} M$. 
In the case of an $\operatorname{ALS}\left(\theta, D,[\cdot, \cdot]_{\theta}\right)$, the presymplectic form $\Omega$ is related with an almost Poisson structure on $R^{*}$, defined and studied below in section 4 .

We recall now a very simple result from the linear algebra of symplectic vector spaces [2, p. 40].

Lemma 2.1. Suppose that $\Omega: V \times V \rightarrow \mathbb{R}$ is a skew symmetric bilinear map which is non-degenerate on the $2 n$-dimensional real vector space $V$. Let $U \subset V$ be a lagrangian subspace of $V$ (i.e. $\Omega_{U \times U}=0$ and $\operatorname{dim} U=n$ ) and $W \subset V$ be another subspace which is a complement to $U$. Then there is a lagrangian vector subspace canonically associated with $W$ and complement to $U$.

If $\theta$ is a symplectic vector bundle, we say that a lagrangian subbundle is a vector subbundle $\mu \subset \theta$ such that, at every point of the base, the fibre of $\mu$ is a lagrangian subspace of the fibre of $\theta$.

It is easy to see that the R-vertical subbundle $V \theta^{*}$ is a lagrangian subbundle of the symplectic bundle $R \theta^{*}$. We say that an R-connection on $\theta^{*}$ is lagrangian if its horizontal subbundle is lagrangian.

Using Lemma 2.1 we can state:

Proposition 2.2. Let $\left(\theta, D,[\cdot, \cdot]_{\theta}\right)$ be an $A L S$ and $C$ be an $R$-connection on $\theta^{*}$. There is an $R$-connection $C^{\prime}$ on $\theta^{*}$, lagrangian with respect to $\Omega$ and canonically associated with $C$.

In the case of the Lie algebroid $\theta=\tau M$ we obtain:

Corollary 2.1. If $C$ is a connection on $\tau^{*} M$, then there is a lagrangian connection $C^{\prime}$ on $\tau^{*} M$, canonically associated with $C$.

For the given vector bundle $\theta=(R, q, M)$ the vertical bundle $V \theta$ is canonically isomorphic with the induced bundle $q^{*} \theta$ and there is a canonical Lie algebroid structure on $V \theta$, where the anchor is the inclusion $V \theta \hookrightarrow \tau R$ and the bracket is induced by the Lie bracket on $\mathcal{X}(R)$. If $f: R \rightarrow \mathbb{R}$ is a real function, then the vertical Hessian of $f$ is defined using local coordinates by:

$$
\operatorname{Hess}_{v}(f)\left(X^{a} \frac{\partial}{\partial y^{a}}, Y^{b} \frac{\partial}{\partial y^{b}}\right) \stackrel{\text { def }}{=} X^{a} Y^{b} \frac{\partial^{2} f}{\partial y^{a} \partial y^{b}} .
$$

Notice that $\operatorname{Hess}_{v}(f)$ is a symmetric bilinear form on the fibres of $V \theta$ ant its definition does not depend on the adapted local coordinates.

A hamiltonian on the vector bundle $\theta^{*}$ is a function $\mathcal{H}: R^{*} \rightarrow \mathbb{R}$ which is differentiable of class $C^{\infty}$ on $\widetilde{R^{*}}=R^{*} \backslash\{0\}$ (where $\{0\}$ is the image of the null section) and continuous on the null section. A regular hamiltonian on $\theta^{*}$ is a hamiltonian $\mathcal{H}$ which has Hess $_{v}(\mathcal{H})$ non-degenerate. According to $[14,15]$, a Hamilton space is a manifold $M$ with a regular hamiltonian $\mathcal{H}$ on the cotangent bundle $\tau^{*} M$. In this case, a canonical connection can be defined on $T^{*} M$.

A lagrangian on the vector bundle $\theta$ is a function $\mathcal{L}: R \rightarrow \mathbb{R}$ which is differentiable of class $C^{\infty}$ on $\widetilde{R}=R \backslash\{0\}$ and continuous on the null section. A regular lagrangian on $\theta$ is a lagrangian $\mathcal{L}$ which has $\operatorname{Hess}_{v}(\mathcal{L})$ non-degenerate. 
Let $\left(\theta, D,[\cdot, \cdot]_{\theta}\right)$ be an ALS and consider a real function $f: R \rightarrow \mathbb{R}$. Using adapted local coordinates, we define a linear vertical form $\omega_{f}$ on $R \theta$ by the formula $\omega_{f}=\frac{\partial f}{\partial y^{\alpha}} \bar{\omega}^{\alpha}$, where $\left\{\omega^{\alpha}, d_{R \theta} y^{\beta}\right\}$ is the dual base (of $\Gamma\left(R^{*} \theta\right)$ ) of the adapted local base $\left\{s_{\alpha}, \frac{\partial}{\partial y^{\alpha}}\right\}$. When the adapted local coordinates change, we have $\bar{\omega}^{\alpha^{\prime}}=g_{\alpha}^{\alpha^{\prime}} \bar{\omega}^{\alpha}$ and $\frac{\partial f}{\partial y^{\alpha^{\prime}}} g_{\alpha}^{\alpha^{\prime}}=\frac{\partial f}{\partial y^{\alpha}}$, thus $\omega_{f}$ is globally defined.

In the sequel we consider only adapted ALS's on $R \theta$.

Proposition 2.3. If $\left(\theta, D,[\cdot, \cdot]_{\theta}\right)$ is an $A L S$ and $\mathcal{L}$ is a lagrangian on $\theta$, then the 2-form $\Omega_{\mathcal{L}}=d_{R \theta} \omega_{\mathcal{L}} \in \mathcal{A}^{2}(R \theta)$ does not depend on the adapted $A L S$ on $R \theta$. If $\mathcal{L}$ is a regular lagrangian, then $\Omega_{\mathcal{L}}$ is non-degenerate, thus $\left(R \theta, \Omega_{\mathcal{L}}\right)$ is a symplectic vector bundle.

Proof. We have:

$$
\begin{aligned}
& \Omega_{\mathcal{L}}=d_{R \theta} \omega_{\mathcal{L}}=d_{R \theta}\left(\frac{\partial \mathcal{L}}{\partial y^{\alpha}}\right) \wedge \omega^{\alpha}+\frac{\partial \mathcal{L}}{\partial y^{\alpha}} d_{R \theta} \omega^{\alpha}= \\
& \left(\frac{\partial^{2} \mathcal{L}}{\partial y^{\beta} \partial y^{\alpha}} d_{R \theta} y^{\beta}+\frac{\partial^{2} \mathcal{L}}{\partial y^{\alpha} \partial x^{i}} D_{\beta}^{i} \omega^{\beta}\right) \wedge \omega^{\alpha}-\frac{1}{2} \frac{\partial \mathcal{L}}{\partial y^{\alpha}} L_{\beta \gamma}^{\alpha} \omega^{\beta} \wedge \omega^{\gamma}= \\
& \frac{\partial^{2} \mathcal{L}}{\partial y^{\beta} \partial y^{\alpha}} d_{R \theta} y^{\beta} \wedge \omega^{\alpha}+\left(\frac{\partial^{2} \mathcal{L}}{\partial y^{\gamma} \partial x^{i}} D_{\beta}^{i}-\frac{\partial^{2} \mathcal{L}}{\partial y^{\beta} \partial x^{i}} D_{\gamma}^{i}-\frac{1}{2} \frac{\partial \mathcal{L}}{\partial y^{\alpha}} L_{\beta \gamma}^{\alpha}\right) \omega^{\beta} \wedge \omega^{\gamma} .
\end{aligned}
$$

Since $\left\{d_{R \theta} y^{\alpha} \wedge d_{R \theta} y^{\beta}, d_{R \theta} y^{\alpha} \wedge \omega^{\beta}, \omega^{\alpha} \wedge \omega^{\beta}\right\}$ is a local base in $\mathcal{A}^{2}(R \theta)$, it follows that $\Omega_{\mathcal{L}}$ is non-degenerate.

In the particular case $\theta=\tau M$, we have $\omega_{\mathcal{L}}=\frac{\partial \mathcal{L}}{\partial y^{i}} d x^{i}$, thus

$$
\Omega_{\mathcal{L}}=d \omega_{\mathcal{L}}=\left(\frac{\partial^{2} \mathcal{L}}{\partial y^{j} \partial y^{i}} d y^{j}+\frac{\partial^{2} \mathcal{L}}{\partial x^{j} \partial y^{i}} d x^{j}\right) \wedge d x^{i}
$$

is the well-known symplectic form on $T M$, defined by the regular lagrangian $\mathcal{L}$. Notice that the vertical subbundle $V \tau M \hookrightarrow \tau T M$ is a lagrangian subbundle related to the symplectic form $\Omega_{\mathcal{L}}$.

Using Lemma 2.1 we can state:

Proposition 2.4. Let $\left(\theta, D,[\cdot, \cdot]_{\theta}\right)$ be an $A L S, \mathcal{L}$ be a regular lagrangian and $C$ be an $R$-connection on $\theta$. There is an $R$-connection $C^{\prime}$ on $\theta$ which is lagrangian with respect to $\Omega_{\mathcal{L}}$ and depends on $C$ and $\mathcal{L}$.

It is well-known that a lagrangian on a manifold gives rise to a canonical connection called the Cartan-Kern connection (see for example [16, 17]). It also known that the horizontal bundle of this connection is also a lagrangian subbundle, thus the Cartan-Kern connection is a lagrangian connection. In the next section we extend these classical results on vector bundles endowed with arbitrary ALS's. It is interesting to remark that the formalism concerning the holonomic mechanics (such as symplectic forms, (semi)sprays, non-linear connections etc.), can be extended in the non-holonomic case of almost Lie structures, too.

3. Canonical R-connections defined by lagrangians and hamiltonians. The aim of this section is to show that a Cartan-Kern R-connection can be associated with 
a lagrangian on an ALS. This connection is a symplectic one and it corresponds to itself when Proposition 2.4 is applied. We define also the Legendre transformation and we show that its R-differential is a symplectic morphism, which sends the Kern-Cartan connection to a symplectic R-connection on the dual.

An $R$-semispray on the $\operatorname{AVB}(\theta, D)$ is a section $S: \widetilde{R}=R \backslash\{0\} \rightarrow R R$ in the both vector bundles of the double vector bundle $(R R, R, R)$, as in diagram (2) for $R=E$; it follows that $t(S)=s(S)$. Using adapted local coordinates, the local form of an Rsemispray $S$ is $\left(x^{i}, y^{\alpha}\right) \rightarrow\left(x^{i}, y^{\alpha}, y^{\alpha}, S^{\beta}\left(x^{i}, y^{\alpha}\right)\right)$. An $R$-spray is an R-semispray which is 2-homogeneous on the fibres of $\widetilde{R}$. An R-(semi)spray is defined by a vector field $\bar{S} \in \mathcal{X}(\tilde{R})$, $\bar{S}: \tilde{R} \rightarrow T \tilde{R}$ such that $q_{*} \circ \bar{S}=\tilde{D}$, where $\tilde{D}$ is the restriction to $\tilde{R}$ of the anchor $D$ and $q: R \rightarrow M$ is the canonical projection of $\theta$. According to [35], the vector field $\bar{S}$ is called an admissible vector field on $R$. Certainly, $S$ and $\bar{S}$ are related by $\bar{S}=\Delta \circ S$, where $\Delta: R \theta \rightarrow \tau R$ is the canonical anchor on $R \theta$. Using $\bar{S}$, an R-spray is defined in [29], where it is called a spray-like.

There is well-known (see [6]) that a semi-spray on a vector bundle defines in a canonical way a non-linear connection on the given vector bundle. Using local coordinates, a more simplified description of this theory is given in $[16,17]$. In an analogous way, in order to avoid a more complicate exposition, we use adapted local coordinates. Despite of this fact, the definitions of the considered objects are free of coordinates.

In the case of an ALS, the classical theory has a natural extension. Thus, an Rsemispray $S$ on an ALS defines canonically an R-connection on $\theta$. More exactly, using local coordinates, if $\left\{S^{\alpha}\left(x^{i}, y^{\beta}\right)\right\}$ are the local components of $S$, then it can be proved, by a straightforward computation, that the local functions $\left\{\frac{1}{2}\left(-\frac{\partial S^{\alpha}}{\partial y^{\beta}}+L_{\beta \gamma}^{\alpha} y^{\gamma}\right)\right\}$ are the local components of an R-connection on $\theta$.

Proposition 3.1. If $\left(\theta, D,[\cdot, \cdot]_{\theta}\right)$ is an $A L S$ and $\mathcal{L}: R \rightarrow \mathbb{R}$ is a non-degenerate lagrangian on $R$, then there is an $R$-connection canonically associated with $\mathcal{L}$ and called the Cartan-Kern R-connection.

Proof. Let us denote by $g_{\alpha \beta}=\frac{\partial^{2} \mathcal{L}}{\partial y^{\alpha} y^{\beta}}$ the local components of the metric tensor on the R-vertical bundle of $\theta$. Notice that these components have a tensorial meaning, since the local sections $\left\{\frac{\partial}{\partial y^{\alpha}}\right\}$ change according to the formula $\frac{\partial}{\partial y^{\alpha}}=g_{\alpha}^{\alpha^{\prime}}\left(x^{i}\right) \frac{\partial}{\partial y^{\alpha^{\prime}}}$.

By a straightforward computation, one proves that

$$
S^{\alpha}=g^{\alpha \beta}\left(-\frac{\partial^{2} \mathcal{L}}{\partial y^{\beta} \partial x^{i}} D_{\gamma}^{i} y^{\gamma}+\frac{\partial \mathcal{L}}{\partial x^{i}} D_{\beta}^{i}-\frac{\partial \mathcal{L}}{\partial y^{\mu}} L_{\beta \gamma}^{\mu} y^{\gamma}\right)
$$

are the components of an R-semispray $S$ on $\theta$. Thus

$$
N_{\beta}^{\alpha}=\frac{1}{2}\left(-\frac{\partial S^{\alpha}}{\partial y^{\beta}}+L_{\beta \gamma}^{\alpha} y^{\gamma}\right)
$$

are the components of an R-connection on $\theta$, canonically associated with $\mathcal{L}$.

In the particular case of a lagrangian on $M$, when $D_{k}^{i}=\delta_{k}^{i}$ and $L_{j k}^{i}=0$, then the R-connection defined above becomes the well-known Cartan-Kern non-linear connection.

Notice also that in the case of a 2 -homogeneous lagrangian (i.e. $\mathcal{L}$ is a Finsler metric) the R-semispray is 2-homogeneous, thus it becomes an R-spray. 
Proposition 3.2. The Cartan-Kern R-connection, defined by a non-degenerate lagrangian $\mathcal{L}$ on $R$, is a symplectic $R$-connection according to the symplectic structure defined on $R \theta$ by $\mathcal{L}$.

Proof. We use adapted local coordinates and we consider a new local base of $\Gamma\left(R \theta^{*}\right)$ (which is adapted to the Cartan-Kern R-connection): $\left\{\bar{\omega}^{\alpha}, \delta_{R \theta} y^{\beta}=d_{R \theta} y^{\beta}+N_{\gamma}^{\beta} \omega^{\gamma}\right\}$. This local base of $\Gamma\left(R^{*} \theta\right)$ is the dual base of the adapted local base $\left\{\bar{s}_{\alpha}=s_{\alpha}-N_{\alpha}^{\beta} \frac{\partial}{\partial y^{\beta}}, \frac{\partial}{\partial y^{\alpha}}\right\}$ of $\Gamma(R \theta)$, where $\left\{N_{\alpha}^{\beta}\right\}$ are the local components of the R-connection $C$. The almost symplectic form $\Omega_{\mathcal{L}}$, is given by:

$$
\begin{aligned}
\Omega_{\mathcal{L}} & =d_{R \theta} \omega_{\mathcal{L}}=d_{R \theta}\left(\frac{\partial \mathcal{L}}{\partial y^{\alpha}}\right) \wedge \bar{\omega}^{\alpha}+\frac{\partial \mathcal{L}}{\partial y^{\alpha}} d_{R \theta} \bar{\omega}^{\alpha} \\
& =\left(\frac{\partial^{2} \mathcal{L}}{\partial y^{\beta} \partial y^{\alpha}} \delta_{R \theta} y^{\beta}+\mathcal{L}_{\beta \alpha} \bar{\omega}^{\beta}\right) \wedge \bar{\omega}^{\alpha}-\frac{1}{2} \frac{\partial \mathcal{L}}{\partial y^{\alpha}} L_{\beta \gamma}^{\alpha} \bar{\omega}^{\beta} \wedge \bar{\omega}^{\gamma} \\
& =\frac{\partial^{2} \mathcal{L}}{\partial y^{\beta} \partial y^{\alpha}} \delta_{R \theta} y^{\beta} \wedge \bar{\omega}^{\alpha}+\frac{1}{2}\left(\mathcal{L}_{\beta \gamma}-\frac{\partial \mathcal{L}}{\partial y^{\alpha}} L_{\beta \gamma}^{\alpha}\right) \bar{\omega}^{\beta} \wedge \bar{\omega}^{\gamma},
\end{aligned}
$$

where

$$
\mathcal{L}_{\gamma \alpha}=\Delta\left(\bar{s}_{\gamma}\right)\left(\frac{\partial \mathcal{L}}{\partial y^{\alpha}}\right)=D_{\gamma}^{i} \frac{\partial}{\partial x^{i}}\left(\frac{\partial \mathcal{L}}{\partial y^{\alpha}}\right)-N_{\gamma}^{\beta} \frac{\partial}{\partial y^{\beta}}\left(\frac{\partial \mathcal{L}}{\partial y^{\alpha}}\right)=D_{\gamma}^{i} \frac{\partial^{2} \mathcal{L}}{\partial x^{i} \partial y^{\alpha}}-N_{\gamma}^{\beta} \frac{\partial^{2} \mathcal{L}}{\partial y^{\beta} \partial y^{\alpha}}
$$

Thus

$$
\Omega_{\mathcal{L}}=d_{R \theta} \omega_{\mathcal{L}}=\frac{\partial^{2} \mathcal{L}}{\partial y^{\beta} \partial y^{\alpha}} \delta_{R \theta} y^{\beta} \wedge \bar{\omega}^{\alpha}+\frac{1}{2} T_{\beta \gamma} \bar{\omega}^{\beta} \wedge \bar{\omega}^{\gamma},
$$

where $T_{\beta \gamma}=D_{\beta}^{i} \frac{\partial^{2} \mathcal{L}}{\partial x^{i} \partial y^{\gamma}}-N_{\beta}^{\nu} \frac{\partial^{2} \mathcal{L}}{\partial y^{\nu} \partial y^{\gamma}}+\frac{1}{2} \frac{\partial \mathcal{L}}{\partial y^{\nu}} L_{\beta \gamma}^{\nu}$.

Using (6) and (7) (which give the components of the Cartan-Kern R-connection), by a straightforward computation one proves that $T_{\beta \alpha}=T_{\alpha \beta}$. Therefore, in the local base $\left\{\delta_{R \theta} y^{\alpha} \wedge \delta_{R \theta} y^{\beta}, \delta_{R \theta} y^{\alpha} \wedge \bar{\omega}^{\beta}, \bar{\omega}^{\alpha} \wedge \bar{\omega}^{\beta}\right\}$ of $\mathcal{A}^{2}(R \theta)$ we have:

$$
\Omega_{\mathcal{L}}=d_{R \theta} \omega_{\mathcal{L}}=\frac{\partial^{2} \mathcal{L}}{\partial y^{\alpha} \partial y^{\beta}} \delta_{R \theta} y^{\alpha} \wedge \bar{\omega}^{\beta} .
$$

Thus the conclusion follows.

The Legendre transformation associated with $\mathcal{L}$ is the function $\mathfrak{L}: \widetilde{R} \rightarrow \widetilde{R^{*}}$ defined by

$$
\mathfrak{L}\left(X^{\alpha} s_{\alpha}\right)=X^{\alpha} \frac{\partial L}{\partial y^{\alpha}} .
$$

Then $\mathfrak{L}$ can be regarded as well as defined on each fibre of $\widetilde{R}$ by $\mathfrak{L}=\frac{\partial \mathcal{L}}{\partial y^{\alpha}} \omega^{\alpha}$, where the local base $\left\{\omega^{\alpha}\right\}$ of $\Gamma\left(\theta^{*}\right)$ is the dual base of the local base $\left\{s_{\alpha}\right\}$ of $\Gamma(\theta)$. It is easy to see that the local form of the Legendre transformation $\mathfrak{L}$ is

$$
\left(x^{i}, y^{\alpha}\right) \rightarrow\left(x^{i}, \frac{\partial \mathcal{L}}{\partial y^{\alpha}}\right) .
$$

Notice that $\mathfrak{L}$ is a fibered manifold morphism which is globally defined, but, generally it fails to comes from a vector bundle morphism.

Taking into account the local form of the Legendre transformation, it follows that the lagrangian $\mathcal{L}$ is regular iff its Legendre transformation $\mathfrak{L}$ is a local diffeomorphism, when $\mathfrak{L}$ induces local diffeomorphisms on each fiber. 
The lagrangian $\mathcal{L}$ is $L$-regular if its Legendre transformation $\mathfrak{L}$ is a global diffeomorphism of $\widetilde{R}$ on $\widetilde{R}^{*}$.

Consider now a hamiltonian $\mathcal{H}$. The Legendre* transformation associated with $\mathcal{H}$ is the function $\mathfrak{H}: \widetilde{R^{*}} \rightarrow \widetilde{R}$ defined by

$$
\mathfrak{H}\left(u_{\alpha} \omega^{\alpha}\right)=u_{\alpha} \frac{\partial \mathcal{H}}{\partial p_{\alpha}} .
$$

It can be regarded as well as defined on each fibre of $\widetilde{R^{*}}$ by $\mathfrak{H}=\frac{\partial \mathcal{H}}{\partial p_{\alpha}} s_{\alpha}$. The local form of the Legendre* transformation $\mathfrak{H}$ is

$$
\left(x^{i}, p_{\alpha}\right) \rightarrow\left(x^{i}, \frac{\partial \mathcal{H}}{\partial p_{\alpha}}\right) .
$$

Notice also that $\mathfrak{H}$ is a fibered manifold morphism, but generally it fails to come from a vector bundle morphism.

Taking into account the local form of the Legendre* transformation, it follows that the hamiltonian $\mathcal{H}$ is regular iff its Legendre* transformation $\mathfrak{H}$ is a local diffeomorphism, when $\mathfrak{H}$ induces local diffeomorphisms on each fibers.

The hamiltonian $\mathcal{H}$ is $H$-regular if its Legendre* transformation $\mathfrak{H}$ is a global diffeomorphism of $\widetilde{R^{*}}$ on $\widetilde{R}$.

As in the classical case, the link between the Lagrange and Hamilton geometry is given by the following result.

Proposition 3.3. a) If $\mathcal{L}: R \rightarrow \mathbb{R}$ is an L-regular lagrangian, then $\mathcal{H}=(Z(\mathcal{L})-$ $\mathcal{L}) \circ \mathfrak{L}^{-1}$ is an $H$-regular hamiltonian on $\theta^{*}$, where $Z \in \mathcal{X}(R)$ is the Liouville vector field and $\mathfrak{L}: R \rightarrow R^{*}$ is the Legendre transformation.

b) If $\mathcal{H}: R^{*} \rightarrow \mathbb{R}$ is an H-regular hamiltonian, then $\mathcal{L}=(\Xi(\mathcal{H})-\mathcal{H}) \circ \mathfrak{H}^{-1}$ is an $L$-regular lagrangian on $\theta$, where $\Xi \in \mathcal{X}\left(R^{*}\right)$ is the Liouville vector field and $\mathfrak{H}: R^{*} \rightarrow R$ is the Legendre* transformation.

Proof. We prove only b), since the proof of a) can be performed in an analogous way. The proof uses classic arguments.

We denote by $\mathfrak{K}=\mathfrak{H}^{-1}$. In adapted local coordinates the local form of $\mathfrak{K}$ is $\left(x^{i}, y^{\alpha}\right) \rightarrow$ $\left(x^{i}, K_{\beta}\left(x^{i}, y^{\alpha}\right)\right)$. The condition $\mathfrak{K} \circ \mathfrak{H}=i d_{\widetilde{R^{*}}}$ has the local form:

$$
K_{\beta}\left(x^{i}, H^{\mu}\left(x^{k}, p_{\gamma}\right)\right)=p_{\beta},
$$

where $H^{\mu}\left(x^{k}, p_{\gamma}\right)=\frac{\partial \mathcal{H}}{\partial p_{\mu}}\left(x^{k}, p_{\gamma}\right)$. Differentiating with respect to $p_{\alpha}$ the relation (10), it follows that $\frac{\partial K_{\beta}}{\partial y^{\delta}}\left(x^{i}, H^{\mu}\left(x^{k}, p_{\gamma}\right)\right) \cdot \frac{\partial H^{\delta}}{\partial p_{\alpha}}\left(x^{k}, p_{\gamma}\right)=\delta_{\beta}^{\alpha}$, or $\frac{\partial K_{\beta}}{\partial y^{\delta}}\left(x^{i}, H^{\mu}\left(x^{k}, p_{\gamma}\right)\right) \cdot h^{\alpha \delta}=\delta_{\beta}^{\alpha}$, where $h^{\alpha \delta}=\frac{\partial^{2} \mathcal{H}}{\partial p_{\alpha} \partial p_{\delta}}$. Denote $\left(h_{\alpha \beta}\right)=\left(h^{\alpha \beta}\right)^{-1}$; then

$$
\frac{\partial K_{\beta}}{\partial y^{\alpha}}\left(x^{i}, H^{j}\left(x^{k}, p_{\gamma}\right)\right)=h_{\alpha \beta}\left(x^{k}, p_{\gamma}\right) .
$$

The Liouville field has the local form $\Xi=p_{\beta} \frac{\partial}{\partial p_{\beta}}$. Using the definition of $\mathfrak{L}$ it follows:

$$
\mathcal{L}\left(x^{i}, y^{\gamma}\right)=K_{\beta}\left(x^{i}, y^{\gamma}\right) \frac{\partial \mathcal{H}}{\partial p_{\beta}}\left(x^{i}, K_{\delta}\left(x^{i}, y^{\gamma}\right)\right)-\mathcal{H}\left(x^{i}, K_{\delta}\left(x^{i}, y^{\gamma}\right)\right)
$$


We have

$$
\frac{\partial \mathcal{L}}{\partial y^{\alpha}}=\frac{\partial K_{\beta}}{\partial y^{\alpha}} \frac{\partial \mathcal{H}}{\partial p_{\beta}}+K_{\beta} \frac{\partial K_{\gamma}}{\partial y^{\alpha}} \frac{\partial^{2} \mathcal{H}}{\partial p_{\gamma} \partial p_{\beta}}-\frac{\partial K_{\beta}}{\partial y^{\alpha}} \frac{\partial \mathcal{H}}{\partial p_{\beta}}=K_{\beta} \frac{\partial K_{\gamma}}{\partial y^{\alpha}} \frac{\partial^{2} \mathcal{H}}{\partial p_{\gamma} \partial p_{\beta}}=K_{\beta} h_{\alpha \gamma} h^{\gamma \beta}=K_{\alpha},
$$
thus using (11) we have $\frac{\partial^{2} \mathcal{L}}{\partial y^{\alpha} \partial y^{\beta}}=\frac{\partial K_{\beta}}{\partial y^{\alpha}}=h_{\alpha \beta}$. It follows that

$$
\frac{\partial^{2} \mathcal{L}}{\partial y^{\alpha} \partial y^{\beta}}\left(x^{i}, y^{\gamma}\right)=h_{\alpha \beta}\left(x^{i}, K_{\delta}\left(x^{i}, y^{\gamma}\right)\right)
$$

In the particular case of a Finsler metric on $\theta$ (i.e. the lagrangian $\mathcal{L}$ is 2-homogeneous), we have $Z(\mathcal{L})=2 \cdot \mathcal{L}$, thus $\mathcal{H}=\mathcal{L} \circ \mathfrak{L}^{-1}$. For a 2 -homogeneous hamiltonian $\mathcal{H}$ on $\theta^{*}$ we obtain a similar result: $\Xi(\mathcal{H})=2 \cdot \mathcal{H}$, thus $\mathcal{L}=\mathcal{H} \circ \mathfrak{H}^{-1}$.

Consider now the Legendre transformation $\mathfrak{L}: \theta \rightarrow \theta^{*}$ (associated with a lagrangian $\mathcal{L})$, viewed as a fibered manifold morphism. Following [25], we can consider the Rdifferential of $\mathfrak{L}$, denoted by $\mathfrak{L}^{T}: R \theta \rightarrow R \theta^{*}$ and given in local coordinates by:

$$
\mathfrak{L}^{T}\left(x^{i}, y^{\alpha}, X^{\beta}, Y^{\gamma}\right)=\left(x^{i}, \frac{\partial \mathcal{L}}{\partial y^{\alpha}}, X^{\beta}, \frac{\partial^{2} \mathcal{L}}{\partial x^{j} \partial y^{\gamma}} D_{\mu}^{j} X^{\mu}+\frac{\partial^{2} \mathcal{L}}{\partial y^{\mu} \partial y^{\gamma}} Y^{\mu}\right) .
$$

A symplectic morphism of two symplectic vector bundles $\xi^{\prime}=\left(E^{\prime}, \pi^{\prime}, M^{\prime}\right)$ and $\xi=$ $(E, \pi, M)$ is a vector bundle morphism $\left(f_{0}, f\right)$, where $f_{0}: M^{\prime} \rightarrow M$ and $f: E^{\prime} \rightarrow E$, which is compatible with the presymplectic forms $\omega^{\prime}$ and $\omega$ respectively, i.e. $\omega_{f_{0}\left(x^{\prime}\right)}^{\prime}\left(f_{x^{\prime}}\left(X^{\prime}\right)\right.$, $\left.f_{x^{\prime}}\left(Y^{\prime}\right)\right)=\omega_{x^{\prime}}^{\prime}\left(X^{\prime}, Y^{\prime}\right),(\forall) x^{\prime} \in M^{\prime}$ and $X^{\prime}, Y^{\prime} \in E_{x^{\prime}}^{\prime}$. It is easy to check the following result.

Proposition 3.4. Let $\left(\theta, D,[\cdot, \cdot]_{\theta}\right)$ be an ALS on $\theta$. Consider the canonical symplectic structure on $R \theta^{*}$ and the symplectic structure on $R \theta$, defined by a regular lagrangian $\mathcal{L}$ on $\theta$. Then the $R$-differential $\mathfrak{L}^{T}: R \theta \rightarrow R \theta^{*}$ of the Legendre transformation of $\mathcal{L}$ is a symplectic morphism.

Now we can prove the following result:

Proposition 3.5. If $\mathcal{H}$ is a regular hamiltonian on $\theta^{*}$, then it defines canonically a lagrangian $R$-connection on $\theta^{*}$.

Proof. According to Proposition 3.3, there is a regular lagrangian $\mathcal{L}$ on $\theta$, canonically associated with $\mathcal{H}$. Let $H \theta \subset R \theta$ be the horizontal subbundle of the Cartan-Kern connection defined by $\mathcal{L}$. Then $H \theta^{*}=\left(\mathfrak{L}^{T}\right)^{-1}(H \theta) \subset R \theta^{*}$ is a horizontal subbundle of an R-connection on $\theta^{*}$. Since $H \theta \subset R \theta$ is a lagrangian subbundle and $\left(\mathfrak{L}^{T}\right)^{-1}$ is a symplectic morphism it follows that $H \theta^{*} \subset R \theta^{*}$ is a lagrangian subbundle.

4. Lagrange's equations for almost Lie structures. In this section, a physical interpretation concerning the constructions performed in the previous sections is given. Using the formalism presented in this paper, we prove that the Lagrange equations for a Lie algebroid, given in [35], keep the same form in the general case of an ALS. In order to emphasize the link between the two approaches, two different proofs of the Lagrange equations are given: the first is a direct one, using the previous constructions, and the second one follows the Weinstein's ideas, using an almost Lagrange Poisson bracket (see [35]). Thus, our construction is a natural extension of the classical case and of the Lie algebroids case, too. 
Let us consider an AVB $(\theta, D)$, where $\theta=(R, q, M)$. According to [35], a tangent vector $X_{y} \in T_{y} R, y \in R$, is admissible if $q_{*, y}(X)=D(y)$. A vector field $X \in \mathcal{X}(R)$ is admissible if it is admissible in every point of $R$. In adapted local coordinates, a vector field $X$ is admissible iff it has the local form $\left(x^{i}, y^{\alpha}\right) \rightarrow\left(x^{i}, y^{\alpha}, D_{\alpha}^{j} y^{\alpha}, F^{\beta}\left(x^{i}, y^{\alpha}\right)\right)$. It is easy to see that $X$ defines on $\theta$ an R-semispray by $\left(x^{i}, y^{\alpha}\right) \rightarrow\left(x^{i}, y^{\alpha}, y^{\alpha}, F^{\beta}\left(x^{i}, y^{\alpha}\right)\right)$. Conversely, an R-semispray $S: R \rightarrow R R$ on $\theta$ defines an admissible vector field $X=\Delta \circ S$, where $\Delta: R \theta \rightarrow \tau R$ is the canonical anchor considered before. Thus, an admissible vector field is an equivalent way to define an R-semispray on an ALS.

Let $S: R \rightarrow R R$ be an R-semispray and $\gamma$ be a integral curve of the corresponding admissible vector field $X=\Delta \circ S$. If $S$ and $\gamma$ have the local forms $\left(x^{i}, y^{\alpha}\right) \rightarrow$ $\left(x^{i}, y^{\alpha}, y^{\alpha}, S^{\beta}\left(x^{i}, y^{\alpha}\right)\right)$ and $t \rightarrow\left(\gamma^{i}(t), u^{\alpha}(t)\right)$ respectively, then the local form of the second order differential equation defined by the semispray $S$ is given by:

$$
\frac{d \gamma^{i}}{d t}=D_{\alpha}^{i}\left(\gamma^{j}\right) u^{\alpha}, \frac{d u^{\alpha}}{d t}=S^{\alpha}\left(\gamma^{j}, u^{\beta}\right)
$$

THEOREM 4.1. Let us consider an $A L S\left(\theta, D,[\cdot, \cdot]_{\theta}\right)$, a regular lagrangian $\mathcal{L}: R \rightarrow \mathbb{R}$ which define the semispray $S$ and an integral curve $\gamma$ of the admissible vector field $X=$ $\Delta \circ S$. If $\gamma$ has the parametrization $t \rightarrow\left(\gamma^{i}(t), u^{\alpha}(t)\right)$, then the Lagrange equation hold:

$$
\frac{d}{d t}\left(\frac{\partial \mathcal{L}}{\partial y^{\alpha}}\left(\gamma^{i}, u^{\beta}\right)\right)=\frac{\partial \mathcal{L}}{\partial x^{i}} D_{\gamma}^{i}-\frac{\partial \mathcal{L}}{\partial y^{\mu}} L_{\alpha \nu}^{\mu} u^{\nu} .
$$

Proof. We have:

$$
\frac{d}{d t}\left(\frac{\partial \mathcal{L}}{\partial y^{\alpha}}\left(\gamma^{i}, u^{\beta}\right)\right)=\frac{\partial^{2} \mathcal{L}}{\partial x^{i} \partial y^{\alpha}} \frac{d \gamma^{i}}{d t}+\frac{\partial^{2} \mathcal{L}}{\partial y^{\beta} \partial y^{\alpha}} \frac{d u^{\beta}}{d t} .
$$

Using the local form (6) of the semispray defined by $\mathcal{L}$ and the equations (12), we obtain:

$$
\begin{aligned}
& \frac{d}{d t}\left(\frac{\partial \mathcal{L}}{\partial y^{\alpha}}\left(\gamma^{i}, u^{\beta}\right)\right)= \\
& \frac{\partial^{2} \mathcal{L}}{\partial x^{i} \partial y^{\alpha}} D_{\beta}^{i} u^{\beta}+g_{\alpha \beta} g^{\beta \gamma}\left(-\frac{\partial^{2} \mathcal{L}}{\partial y^{\gamma} \partial x^{i}} D_{\mu}^{i} u^{\mu}+\frac{\partial \mathcal{L}}{\partial x^{i}} D_{\gamma}^{i}-\frac{\partial \mathcal{L}}{\partial y^{\mu}} L_{\beta \nu}^{\mu} u^{\nu}\right) .
\end{aligned}
$$

Thus the Lagrange equation (13) holds.

In the particular case of a Lie algebroid, the Lagrange equation (13) becomes the Lagrange equation (2) from [35]. Notice that the Lagrange equation (13) is meaningful even the lagrangian is not regular.

The Weinstein proof of Lagrange equation uses the Poisson bracket on the dual of the Lie algebroid. It is interesting that this method can be used in order to prove the Lagrange equation on an ALS, without major modifications, using instead an almost Poisson bracket.

Let us call an almost Poisson bracket on a manifold $M$ a skew-symmetric and bilinear $\mathbb{R}$-map $\{\cdot, \cdot\}: \mathcal{F}(M) \times \mathcal{F}(M) \rightarrow \mathcal{F}(M)$, which satisfies the Leibniz rule:

$$
\left\{f, f^{\prime} \cdot f^{\prime \prime}\right\}=\left\{f, f^{\prime}\right\} \cdot f^{\prime \prime}+f^{\prime} \cdot\left\{f, f^{\prime \prime}\right\},(\forall) f, f^{\prime}, f^{\prime \prime} \in \mathcal{F}(M) .
$$

The Leibniz rule associates with every function $f \in \mathcal{F}(M)$ a vector field $X_{f} \in \mathcal{X}(M)$, called the almost hamiltonian vector field of $f$. If the bracket satisfy the Jacobi condition, then it is the well-known Poisson bracket. 
If $\left(\theta, D,[\cdot, \cdot]_{\theta}\right)$ is a Lie algebroid, then a Poisson bracket can be defined on the dual $R^{*}$. Every $f \in \mathcal{F}(M)$ and $s \in \Gamma(\theta)$ lift to $\tilde{f}, \tilde{s} \in \mathcal{F}\left(R^{*}\right)$, where $\tilde{f}=q^{*} f$ and $\tilde{s}(\omega)=\omega(s)$, $(\forall) \omega \in R^{*}$. According to [1], the Poisson bracket is defined by:

$$
\left\{\tilde{f}, \tilde{f}^{\prime}\right\}=0,\{\tilde{s}, \tilde{f}\}=\widetilde{D(s)(f)},\left\{\tilde{s}, \tilde{s}^{\prime}\right\}=-\widetilde{\left[s, s^{\prime}\right]_{\theta}},(\forall) f, f^{\prime} \in \mathcal{F}(M), s, s^{\prime} \in \Gamma(\theta)
$$

(see also $[4,5,35])$. The local form of the bracket is given by:

$$
\left\{x^{i}, x^{j}\right\}=0,\left\{x^{i}, p_{\alpha}\right\}=D_{\alpha}^{i},\left\{p_{\alpha}, p_{\beta}\right\}=-L_{\alpha \beta}^{\gamma} p_{\gamma},
$$

where $\left(D_{\alpha}^{i}\right)$ and $\left(L_{\alpha \beta}^{\gamma}\right)$ are the local components of the anchor $D$ and of the bracket $[\cdot, \cdot]_{\theta}$ respectively.

Let $\left(\theta, D,[\cdot, \cdot]_{\theta}\right)$ be an ALS. Using the Leibniz rule, the formulas (14) extend to a map $\{\cdot, \cdot\}: \mathcal{F}\left(R^{*}\right) \times \mathcal{F}\left(R^{*}\right) \rightarrow \mathcal{F}\left(R^{*}\right)$, which is an almost Poisson bracket on $R^{*}$. Notice that this almost Poisson bracket is a Poisson bracket on $R^{*}$ iff $\left(\theta, D,[\cdot, \cdot]_{\theta}\right)$ is a Lie algebroid. Using the presymplectic form $\Omega$, given by formula (5), the bracket given by (14) can be defined also as follows. Since the form $\Omega$ is non-degenerate on the fibers of $R \theta^{*}$, its inverse on each fiber defines an isomorphism of the vector bundles $R \theta^{*}$ and $\left(R \theta^{*}\right)^{*}=R \theta$, denoted as $\# \Omega$. If $h^{\prime}, h^{\prime \prime} \in \mathcal{F}\left(R^{*}\right)$, then

$$
\left\{h^{\prime}, h^{\prime \prime}\right\}=\Omega\left(\#_{\Omega}\left(d_{R \theta^{*}} h^{\prime}\right), \#_{\Omega}\left(d_{R \theta^{*}} h^{\prime \prime}\right)\right) .
$$

Consider a regular lagrangian $\mathcal{L}: R \rightarrow \mathbb{R}$. An almost Lagrange Poisson structure on $R$, which pulls back the almost Poisson structure on $R^{*}$ to an almost Poisson structure on $R$, can be defined. This can be done using the bracket relations:

$$
\left\{x^{i}, x^{j}\right\}=0,\left\{x^{i}, \frac{\partial \mathcal{L}}{\partial y^{\alpha}}\right\}=D_{\alpha}^{i},\left\{\frac{\partial \mathcal{L}}{\partial y^{\alpha}}, \frac{\partial \mathcal{L}}{\partial y^{\beta}}\right\}=-L_{\alpha \beta}^{\gamma} \frac{\partial \mathcal{L}}{\partial y^{\gamma}}
$$

which use the Legendre transformation and the Leibniz rule.

The action $A$ is the real function on $R$, defined by $A(y)=<\mathfrak{L}(y), y>$, where $\mathcal{L}$ is the Legendre transformation. The energy $E$ is $E=A-L$. In adapted local coordinates, $A=y^{i} \frac{\partial \mathcal{L}}{\partial y^{i}}$ and $E=y^{i} \frac{\partial \mathcal{L}}{\partial y^{i}}-\mathcal{L}$. The almost lagrangian vector field associated with the regular lagrangian $\mathcal{L}$ is the almost hamiltonian lagrangian of the energy $E$. The almost lagrangian vector field $X_{E}$ has the local form $\left\{x^{i}, E\right\} \frac{\partial}{\partial x^{i}}+\left\{y^{\alpha}, E\right\} \frac{\partial}{\partial y^{\alpha}}$. If $\gamma$ is an integral curve of this vector field, which has the local form $t \rightarrow\left(\gamma^{i}(t), u^{\alpha}(t)\right)$, then the following equations follow:

$$
\left\{\begin{aligned}
\frac{d \gamma^{i}}{d t^{\alpha}} & =\left\{x^{i}, E\right\}, \\
\frac{d u^{\alpha}}{d t} & =\left\{y^{\alpha}, E\right\} .
\end{aligned}\right.
$$

Let us prove that the almost lagrangian vector field is admissible. Indeed, using the first equation (17) and the bracket formula $\left\{x^{i}, \mathcal{L}\right\}=\left\{x^{i}, y^{\alpha}\right\} \frac{\partial \mathcal{L}}{\partial y^{\alpha}}$, we have:

$$
\begin{aligned}
\frac{d \gamma^{i}}{d t} & =\left\{x^{i}, E\right\}=\left\{x^{i}, u^{\alpha} \frac{\partial \mathcal{L}}{\partial y^{\alpha}}-\mathcal{L}\right\} \\
& =\left\{x^{i}, y^{\alpha}\right\} \frac{\partial \mathcal{L}}{\partial y^{\alpha}}+\left\{x^{i}, \frac{\partial \mathcal{L}}{\partial y^{\alpha}}\right\} u^{\alpha}-\left\{x^{i}, \mathcal{L}\right\}= \\
& =\left\{x^{i}, \frac{\partial \mathcal{L}}{\partial y^{\alpha}}\right\} u^{\alpha}=D_{\alpha}^{i} u^{\alpha},
\end{aligned}
$$

thus the vector field is admissible. 
Let us prove the Lagrange equation (13) using the almost Lagrange Poisson bracket. We have along $\gamma$ :

$$
\begin{aligned}
\frac{d}{d t}\left(\frac{d \mathcal{L}}{d y^{\alpha}}\right)= & \left\{\frac{d \mathcal{L}}{d y^{\alpha}}, E\right\}=\left\{\frac{d \mathcal{L}}{d y^{\alpha}}, y^{\beta} \frac{\partial \mathcal{L}}{\partial y^{\beta}}-\mathcal{L}\right\} \\
= & \left\{\frac{d \mathcal{L}}{d y^{\alpha}}, y^{\beta}\right\} \frac{\partial \mathcal{L}}{\partial y^{\beta}}+u^{\beta}\left\{\frac{d \mathcal{L}}{d y^{\alpha}}, \frac{\partial \mathcal{L}}{\partial y^{\beta}}\right\}-\left\{\frac{d \mathcal{L}}{d y^{\alpha}}, \mathcal{L}\right\} \\
= & \left\{\frac{d \mathcal{L}}{d y^{\alpha}}, y^{\beta}\right\} \frac{\partial \mathcal{L}}{\partial y^{\beta}}-u^{\beta} L_{\alpha \beta}^{\mu} \frac{d \mathcal{L}}{d y^{\mu}} \\
& -\left\{\frac{d \mathcal{L}}{d y^{\alpha}}, x^{i}\right\} \frac{d \mathcal{L}}{d x^{i}}-\left\{\frac{d \mathcal{L}}{d y^{\alpha}}, y^{\beta}\right\} \frac{d \mathcal{L}}{d y^{\beta}} \\
= & D_{\alpha}^{i} \frac{d \mathcal{L}}{d x^{i}}-u^{\beta} L_{\alpha \beta}^{\mu} \frac{d \mathcal{L}}{d y^{\mu}} .
\end{aligned}
$$

5. Homogeneous connections and R-sprays. A result from [29], which, in turn, generalizes a theorem of Ambrose-Palais-Singer, is extended in this section. It refers to the possibility to define an R-spray using a suitable homogeneous non-linear connection, in the case when the anchor is injective on fibres. In [29] the R-spray $S$ is differentiable on the entire $R$, while in our paper $S$ is differentiable on $\tilde{R}=R \backslash\{0\}$.

Let $(\theta, D)$ be a given AVB. In the sequel we denote by $\tilde{\theta}=(\tilde{R}, \tilde{q}, M)$ the fibered manifold obtained in an obvious way from $\tilde{R}$.

A connection on $\theta$ is a left splitting $C: \tau \tilde{R} \rightarrow V \tilde{\theta}$ of the inclusion $\tilde{\imath}: V \tilde{\theta} \rightarrow \tau \tilde{R}$; it is homogeneous if it is compatible with the homothety on the fibers of $\tilde{R}$. For example the R-spray can be defined by a Finsler metric $\mathcal{L}: R \rightarrow \mathbb{R}$, which is continuous on $R$ and it is smooth on $\tilde{R}$.

If $C$ and $C^{\prime}$ are two homogeneous connections, then there is a vector bundle map $m: \tilde{q}^{*} \tau M \rightarrow V \tilde{\theta}$, which is 1-homogeneous on the fibers of $\tilde{R}$ (we say that $m$ is a homogeneous vector bundle morphism), such that $C^{\prime}-C=m \circ \tilde{\Pi}$, where $\tilde{\Pi}: \tau \tilde{R} \rightarrow \tilde{q}^{*} \tau M$ is the canonical projection. Conversely, if $m: \tilde{q}^{*} \tau M \rightarrow V \tilde{\theta}$ is a homogeneous vector bundle morphism and $C: \tau \tilde{R} \rightarrow V \tilde{\theta}$ is a homogeneous connection, then $C^{\prime}=C+m \circ \tilde{\Pi}$ is also a homogeneous connection.

If $C$ is a homogeneous connection and $S: \tilde{R} \rightarrow R \tilde{R}$ is an R-spray then $\beta=C \circ \Delta \circ S$ : $\tilde{R} \rightarrow V \tilde{R}$ is 2-homogeneous on the fibers of $\tilde{R}$ (i.e. $C \circ \Delta \circ S(\lambda X)=\lambda^{2} C \circ \Delta \circ S(X)$, $(\forall) \lambda>0, X \in \Gamma(\theta))$. If $\beta=0$, then, according to [29], we say that the R-spray $S$ is geodesic related to the homogeneous connection $C$. Notice that for every homogeneous connection $C$ on $\theta$ there is a geodesic R-spray related to $C$. If the anchor $D$ is injective on fibres, then a similar relation between the integral curves of the regular distribution defined by the image of $D$ and the geodesics of the geodesic R-spray $S$, as performed in [29], can be done. (See also [30] for a similar situation.) We do not use any curve here, but we made this observation in order to motivate the name geodesic given to $S$.

A 2-homogeneous map $\beta: \tilde{R} \rightarrow V \tilde{R}$ defines a homogeneous vector bundle morphism $\tilde{\beta}: V \tilde{R} \rightarrow V \tilde{R}$ (i.e. it is homogeneous on the fibres of $\tilde{R}$ ), according to the formulas $\left(x^{i}, y^{a}, Y^{b}\right) \rightarrow\left(x^{i}, y^{a}, \frac{1}{2} \frac{\partial^{2} \beta^{b}}{\partial y^{c} \partial y^{d}} y^{c} Y^{d}\right)$, where $\left(x^{i}, y^{a}\right) \rightarrow\left(x^{i}, y^{a}, \beta^{a}\left(x^{i}, y^{a}\right)\right)$ is the local 
form of $\beta$; the 2-homogeneity of $\beta$ implies that the local functions $\left\{\frac{\partial^{2} \beta^{b}}{\partial y^{c} \partial y^{d}}\left(x^{i}, y^{a}\right)\right\}$ are the local components of a bilinear map $V \tilde{R} \otimes V \tilde{R} \rightarrow V \tilde{R}$. We have that $\tilde{\beta}$ is related to $\beta$ by $\beta=\tilde{\beta}(Z)$, where $Z$ is the Liouville vector field.

Notice that a linear connection on $\theta$ defines and it is defined by a associated homogeneous connection $C: \tau R \rightarrow V \theta$.

The following result generalizes a Theorem of Ambrose-Palais-Singer.

THEOREM 5.1 [29]. Assume that the anchor map is injective on fibers. Then for every $R$-spray $S: R \rightarrow R R$ there exists a linear connection on $\theta$ such that $S$ is the geodesic $R$-spray related to the associated homogeneous connection.

In fact, taking into account the restrictive conditions of this theorem, the R-spray $S$ defines and it is defined by a linear R-connection on $\theta$. The above Theorem can be improved as follows.

THEOREM 5.2. Assume that the anchor map is injective on fibers. Then for every $R$-spray $S: \tilde{R} \rightarrow R \tilde{R}$ there exists a homogeneous connection $C$ on $\theta$, such that $S$ is the geodesic $R$-spray related to $C$.

Proof. Since the anchor $D: \theta \rightarrow \tau M$ is injective on fibers, there is a left splitting $F: \tau M \rightarrow \theta$, i.e. $F \circ D=i d_{R}$, where $\theta=(R, q, M)$. Thus $q^{*} F: q^{*} \tau M \rightarrow q^{*} \theta \cong V \theta$ is a left splitting of $D^{*}: V \theta \rightarrow q^{*} \tau M$. Consider now an arbitrary homogeneous connection $C^{\prime}: \tau \tilde{R} \rightarrow V \tilde{\theta}$ (for example $C^{\prime}$ can be defined by a linear connection on $\theta$ ). Thus, according to some observations made in the beginning of this section, $\beta=C^{\prime} \circ \Delta \circ S$ is 2-homogeneous on the fibers of $\tilde{R}$ and it defines a homogeneous vector bundle morphism $\tilde{\beta}: V \tilde{R} \rightarrow V \tilde{R}$ such that $\beta=\tilde{\beta}(Z)$, where $Z$ is the Liouville vector field. Then $C=$ $C^{\prime}-\tilde{\beta} \circ\left(q^{*} F\right) \circ \tilde{\Pi}$ defines a homogeneous connection on $\theta$, where $\tilde{\Pi}: \tau \tilde{R} \rightarrow \tilde{q}^{*} \tau M$ is the canonical projection. Since $\left(q^{*} F\right) \circ \tilde{\Pi} \circ \Delta \circ S=\left(q^{*} F\right) \circ q^{*} D \circ Z=Z$ and $\tilde{\beta}(Z)=\beta$, it follows that $C \circ \Delta \circ S=C^{\prime} \circ \Delta \circ S-\tilde{\beta} \circ\left(q^{*} F\right) \circ \tilde{\Pi} \circ \Delta \circ S=C^{\prime} \circ \Delta \circ S-\beta=0$, thus $S$ is a geodesic R-spray related to $C$.

Acknowledgments. The authors thank the organizers of the Conference, especially Professor Jan Kubarski, for the kind invitation and their support.

\section{References}

[1] P. Dazord and D. Sondaz, Variétés de Poisson-Algébrö̈des de Lie, Publ. Dept. Math. Lyon I, 1/B(1988), 1-69.

[2] A. Cannas da Silva, Lectures on Symplectic Geometry, University of California at Berkeley, November 30, 1998.

[3] A. Cannas da Silva and A. Weinstein, Geometric Models for Noncommutative Algebras, Berkeley Mathematics Lecture Notes, 10, AMS, Providence, RI, 1999.

[4] R. L. Fernandes, Lie algebroids, holonomy and characteristic classes, math-DG 0007132.

[5] J. Grabowski and P. Urbański, Algebroids-general differential calculi on vector bundles, J. Geom. Phys. 31 (1999), 111-141; math DG/9908174. 
[6] J. Grifone, Structure presque-tangente et connections, Ann. Inst. Fourier (Grenoble) (I): 22, 1 (1972), 287-334; (II): 22, 1 (1972), 291-338.

[7] C. M. Marle, Reduction of constrained mechanical systems and stability of relative equilibria, Comm. Math. Phys. 174 (1995), 296-318.

[8] P. J. Higgins and K. C. H. Mackenzie, Duality for base-changing morphisms of vector bundles, modules, Lie algebroids and Poisson structures, Math. Proc. Camb. Phil. Soc. 114 (1993), 471-488.

[9] K. Konieczna and P. Urbański, Double vector bundles and duality, dg-ga/9710014.

[10] P. Libermann, Lie algebroids and mechanics, Archivum Mathematicum (Brno) 32 (1996), 147-162.

[11] Z.-L. Liu, A. Weinstein and P. Xu, Manin triples for Lie algebroids, J. Diff. Geom. 45 (1997), 547-574; dg-ga/9508013.

[12] K. C. H. Mackenzie, Double Lie algebroids and second order geometry I, Adv. Math. 94 (1992), 180-239.

[13] K. C. H. Mackenzie, Lie algebroids and Lie pseudoalgebras, Bull. London Math. Soc. 27 (1995), 97-147.

[14] R. Miron, Sur la géométrie des espaces d'Hamilton, C.R. Acad. Sci. Paris 306 (1988), 195-198.

[15] R. Miron, The geometry of Hamilton spaces, The Proceedings of the fifth National Seminar on Finsler and Lagrange spaces, Braşov, 1988.

[16] R. Miron and M. Anastasiei, Vector Bundles. Lagrange Spaces. Applications to the Theory of Relativity, Ed. Academiei, Bucureşti, 1987.

[17] R. Miron and M. Anastasiei, The Geometry of Lagrange Spaces: Theory and Applications, Kluwer Acad. Publ., 1994.

[18] R. Miron and A. Bejancu, A new method in geometry of Finsler subspaces, An. St. Univ. "Al. I. Cuza" Iaşi 30, 4 (1984), 56-60.

[19] R. Montgomery, M. Shapiro and A. Stolin, A nonintegrable sub-Riemannian geodesic flow on a Carnot group, J. Dyn. Control Syst. 3 (1997), 519-530.

[20] F. Nuebel, On integral manifolds for vector space distributions, Math. Ann. 294 (1992), $1-17$.

[21] M. Popescu, On almost holonomic structures, Analele Univ. din Craiova, seria mat.-inf. 19 (1991-92), 93-97.

[22] M. Popescu, Semisprays induced on submanifolds, in: New Developments in Diff. Geom., Kluwer Academic Publ., 1998, 317-328.

[23] P. Popescu, On the geometry of relative tangent spaces, Rev. Roum. Math. Pures Appl. 37 (1992), 727-733.

[24] P. Popescu, Almost Lie structures, derivations and R-curvature on relative tangent spaces, Rev. Roum. Math. Pures Appl. 37 (1992), 779-789.

[25] P. Popescu, On quasi-connections on fibered manifolds, in: New Developments in Diff. Geom., Kluwer Academic Publ., 1996, 343-352.

[26] P. Popescu, Categories of modules with differentials, J. Algebra 185 (1996), 50-73.

[27] P. Popescu, On generalized algebroids, in: New Developments in Diff. Geom., Kluwer Academic Publ., 1998, 329-342.

[28] J. Pradines, Géométrie différentielle au-dessus d'un groupoïde, C. R. Acad. Sci. Paris, Série A 266 (1968), 1194-1196.

[29] H. Reckziegel, Generalized sprays and the theorem of Ambrose-Palais-Singer, in: Dillen, Franki et al. (ed.), Geometry and Topology of Submanifolds, V. Proceedings of the con- 
ference on "Theory of submanifolds" (Leuven and Brussels, July 1992), World Scientific, Singapore, 1993, 242-248.

[30] R. Strichartz, Sub-Riemannian geometry, J. Diff. Geom. 24 (1986), 221-263.

[31] I. Vaisman, Lectures on symplectic and Poisson geometry (Notes edited by Fani Petalidou), Summer School on Differential Geometry, Coimbra, September 1999, Textos de Mathemática, série B, Dep. de Mat. da Univ. de Coimbra, Portugal, 2000.

[32] Gh. Vrănceanu, Sur quelques tenseurs dans les variétés non holonomes, C.R. Acad. Sci. Paris 186 (1929), 995.

[33] Y.-C. Wong, Linear connections and quasi connections on a differentiable manifold, Tôhoku Math J. 14 (1962), 49-63.

[34] P. Xu, Gerstenhaber algebras and BV algebras in Poisson geometry, Commun. Math. Phys. 200 (1999), 545-560; dg-ga/9703001.

[35] A. Weinstein, Lagrangian mechanics and groupoids, in: Proceedings of Mechanics Day, Fields Institute. 